\title{
Análise do perfil epidemiológico e da qualidade de vida de pacientes hemodialíticos de um centro nefrológico da cidade de Araguari, Minas
} Gerais.

DOI: $10.47224 /$ rm.v5i10.101

\author{
Arthur Carvalho Faria \\ Ana Paula Espíndola Tamarindo \\ Bruno Miranda de Jesus \\ Jhonatan Pereira Castro \\ Lauana Castro Faria \\ Lincoln Rodrigues Fernandes Júnior \\ Manoelina Louize Queiroz dos Santos \\ Luiz Cláudio Pádua Netto \\ Lucivânia Marques Pacheco
}

e-mail: arthurcarvalhofaria@hotmail.com

\begin{abstract}
Resumo
A doença renal crônica (DRC) consiste em lesões que levam à perda progressiva e irreversível da função dos rins, tendo como principal tratamento a diálise do sangue, processo que implica em realizar mecanicamente a função dos rins até que um transplante seja feito. A DRC acarreta uma série de desarranjos bioquímicos, clínicos e metabólicos, que se somam a repercussões psicossociais, constituindo-se como estressores para os pacientes, podendo causar impacto em sua qualidade de vida (QV). OBJETIVO: Analisar perfil epidemiológico e a qualidade de vida (QV) de pacientes que realizam hemodiálise em um centro nefrológico da cidade de Araguari. METODOLOGIA: Trata-se de um estudo analítico observacional do tipo corte transversal, com abordagem quantitativa, realizado em um centro nefrológico da cidade de Araguari, por meio do sistema NEFRODATA, do questionário SF-36 versão brasileira e do software Excel ${ }^{\circledR}$ e Bioestat, versão 5.0. RESULTADOS: o estudo revelou prevalência das patologias de base Diabetes Mellitus (DM), doença mais prevalente, estando presente em 39 dos 93 pacientes analisados: sendo que 37 pacientes possuíam DM de forma isolada e 2 possuíam DM e Hipertensão arterial Sistêmica (HAS). Já no requisito Qualidade de Vida (QV), o estudo demonstrou que a capacidade funcional dos pacientes com DRC interfere nos aspectos sociais, físicos, mentais e emocionais. CONCLUSÃO: A DRC cresce e crescerá virtuosamente com o aumento da pobreza. Deve-se investir em pesquisas que tragam melhorias para portadores de DRC e outras doenças crônicas.
\end{abstract}

Palavras-chave: Diálise renal, Qualidade de vida, Depressão, Perfil de saúde

\begin{abstract}
Chronic kidney disease (CKD) consists of lesions that lead to progressive and irreversible loss of kidney function. Blood dialysis is the main treatment, a process that involves mechanically performing kidney function until a transplant is performed. CKD entails a series of biochemical, clinical and metabolic disorders, which add to the psychosocial repercussions, constituting stressors for patients, which may affect their quality of life (QL). OBJECTIVE: To analyze the epidemiological profile and quality of life (QL) of hemodialysis patients in a nephrological center in the city of Araguari. METODOLOGY: This is a cross-sectional observational analytical study, with quantitative character, conducted in a nephrological center in the city of Araguari, using the NEFRODATA system, the SF-36 Brazilian version and Excel ${ }^{\circledR}$ and Bioestat software, version 5.0. RESULTS: The study revealed a prevalence of the beginning pathologies Diabetes Mellitus (DM), the most prevalent disease, being present in 39 of the 93 patients analyzed: 37 patients had only DM and 2 had DM and Systemic Hypertension (SH). As for the Quality of Life (QL) requirement, the study demonstrated that the functional capacity of patients with CKD interferes with social, physical, mental and emotional aspects. CONCLUSION: CKD grows and will grow virtuously with increasing poverty. It is necessary to invest in research that will improve the QL of patients with CKD and other chronic diseases.
\end{abstract}

Keywords: Renal dialysis; Quality of life; Depression; Health profile 
Os rins exercem múltiplas funções que podem ser didaticamente caracterizadas como filtração, reabsorção, homeostase, funções endocrinológica e metabólica. A função primordial dos rins é a manutenção da homeostasia, regulando o meio interno, predominantemente pela reabsorção de substâncias e íons filtrados nos glomérulos e excreção de outras substâncias (SODRÉ; COSTA; LIMA, 2007). A doença renal crônica (DRC) consiste em lesão renal e perda progressiva e irreversível da função dos rins (glomerular, tubular e endócrina). A fase terminal (DRCT) corresponde à faixa de função renal na qual os rins perderam o controle do meio interno (CRAVO et al., 2011).

O tratamento definitivo indicado é o transplante renal. Trata-se de um processo moroso, e até que ele se concretize, a única alternativa para manter a vida, está no tratamento dialítico contínuo (TRENTINI et al., 2004). A hemodiálise é o método que consiste na filtração dos líquidos extra corporais do sangue por uma máquina que substitui as funções renais (CAVALCANTE et al., 2011). O número de pessoas em tratamento dialítico, apesar de crescente, manteve-se estável nas últimas avaliações (SCHWARTZ et al., 2017). Somente na modalidade hemodiálise era de 91.475 pessoas em 2008, e passou para 97.586 pessoas em 2012, e em julho de 2014, alcançou o número total estimado de 112.004 pessoas (GONÇALVES et.al, 2015). Segundo a Sociedade Brasileira de Nefrologia, número total de centros ativos de diálise aumentou 37,8\%, de 550 em 2002 para 758 em 2017, enquanto o número de pacientes aumentou 159,4\% no mesmo período (THOMÉ et.al, 2019).

Sabe-se que a população com DRCT tem aumentado nos últimos anos, principalmente devido ao envelhecimento da população geral e ao aumento no número de pacientes com hipertensão arterial e diabetes mellitus (KUSUMOTO et al., 2007). A hipertensão arterial é uma das principais causas de insuficiência renal crônica e a associação dessas duas situações clínicas aumenta consideravelmente o risco cardiovascular. Os principais mecanismos da hipertensão arterial na insuficiência renal crônica são sobrecarga salina e de volume, além de aumento de atividade do sistema renina-angiotensina-aldosterona (SRAA) e disfunção endotelial (BORTOLOTTO, 2008).

A disfunção renal relacionada ao diabetes resulta da interação de diversos fatores: genéticos, ambientais, metabólicos e hemodinâmicos, que, atuando em conjunto, promovem o enfraquecimento da membrana basal glomerular, a expansão da matriz mesangial, a diminuição do número de podócitos, glomeruloesclerose e fibrose tubulointersticial. (MOREIRA et al., 2008).

O paciente renal crônico em hemodiálise convive constantemente com a negação e as consequências da evolução da doença, além de um tratamento doloroso e com as limitações e alterações que repercutem na sua própria qualidade de vida (FRAZÃO; RAMOS; LIRA, 2011). O paciente com DRC, em programa de hemodiálise, é obrigado a conviver diariamente com uma doença incurável que o obriga a uma forma de tratamento dolorosa, de longa duração e que provoca, juntamente com a evolução da doença e suas complicações, ainda maiores limitações e alterações de grande impacto, que repercutem tanto na sua própria qualidade de vida quanto na do grupo familiar (HIGA et al., 2007).

Esses pacientes, que dependem de tecnologia avançada para sobreviver, apresentam limitações no seu cotidiano e vivenciam inúmeras perdas e mudanças biopsicossociais que interferem na sua qualidade de vida tais como: a perda do emprego, alterações na imagem corporal, restrições dietéticas e hídricas (MARTINS; CESARINO, 2005). Humor depressivo persistente, autoimagem prejudicada e sentimentos pessimistas são algumas manifestações psicológicas em pacientes portadores de DRC. (HIGA et al., 2007).

A análise da QV desses pacientes justifica-se na medida em que as limitações bioquímicas, clínicas e metabólicos podem aumentar os riscos de morbidade e mortalidade, agravando as repercussões psicossociais e impactando na $\mathrm{QV}$ do doente. A avaliação da QV relacionada ao tratamento da DCR pode identificar aspectos que requerem maior atenção da equipe multiprofissional de saúde, dando subsídios para a implementação de novas estratégias e melhora da sobrevida dos pacientes. Além disso, apesar do crescente número de pesquisas envolvendo esta temática, faltam dados relativos à cidade de Araguari, o que justifica a realização deste estudo.

Já a análise do perfil epidemiológico dos pacientes com DRC é importante para promoção de melhorias na assistência à saúde desses pacientes, pois possibilita que se trace estratégias de cuidados em saúde com vistas à redução das taxas de morbidade e mortalidade.

A partir da premissa de que limitações bioquímicas, clínicas e metabólicos agravam as repercussões psicossociais, podendo impactar na QV dos pacientes portadores de DRC, observa-se a necessidade de 
analisar o perfil e a qualidade de vida de pacientes de hemodiálise da cidade de Araguari, com vistas à melhoria do tratamento. O objetivo deste estudo é o de analisar perfil epidemiológico e a qualidade de vida (QV) de pacientes que realizam hemodiálise em um centro nefrológico da cidade de Araguari

\section{MATERIAIS E MÉTODOS}

Trata-se de um estudo observacional analítico do tipo corte transversal, com abordagem quantitativa, a ser realizado em um Instituto de Nefrologia do município de Araguari, Minas Gerais.

A população da pesquisa é composta por 93 pacientes do Instituto. Trata-se de uma amostra nãoprobabilística cujos dados foram coletados no segundo semestre de 2019. Foram inclusos na pesquisa todos os prontuários de homens e mulheres que realizaram hemodiálise no Instituto de Nefrologia no período pesquisado. Por se tratar de prontuário eletrônico, não é necessário excluir prontuários por ilegibilidade e falta de informações nos campos.

Para responder ao questionário, foram inclusos todos os pacientes do Instituto no período pesquisado e que concordarem em realizar a pesquisa e como critério de exclusão foi considerado apenas os pacientes que se recusaram a responder o questionário aplicado ou não haviam condições físicas ou psicológicas de transmitirem as informações solicitadas

Os riscos deste estudo são mínimos e referem-se ao constrangimento dos pacientes em relação às perguntas do questionário. Para sanar este risco os pesquisadores garantiram total sigilo da identidade dos pacientes e, para problemas mais graves, como por exemplo algum sintoma de depressão, foi disponibilizado atendimento psicológico pelo setor NAAP do IMEPAC, situado no Avenida Minas Gerais, número 1889. Os dias e horários de funcionamento são de segunda à sexta-feira, das 9 às $11 \mathrm{~h}$ e das 13 às $18 \mathrm{~h}$. Todos os participantes do estudo assinaram o TCLE.

O acesso aos prontuários dos pacientes foi feito por meio do Sistema informatizado utilizado pelo Instituto o qual possui dados já organizados (NEFRODATA).

A análise da QV foi feita por meio do questionário SF36 versão brasileira. O SF-36 é um instrumento genérico de avaliação da qualidade de vida, de fácil administração e compreensão. Consiste em um questionário multidimensional formado por 36 itens, englobados em 8 escalas ou domínios, que são: capacidade funcional, aspectos físicos, dor, estado geral da saúde, vitalidade, aspectos sociais, aspectos emocionais e saúde mental. Apresenta um escore final de 0 (zero) a 100 (obtido por meio de cálculo do Raw Scale), em que o zero corresponde ao pior estado geral de saúde e o 100 corresponde ao melhor estado de saúde.

Serão consideradas as variáveis demográficas: sexo, idade, renda, raça/etnia e escolaridade dos pacientes. Para compor os dados epidemiológicos foram analisados prontuários eletrônicos da população pesquisada e esta respondeu, também, ao questionamento sobre se já havia realizado acompanhamento psicológico ou feito uso de algum tipo de antidepressivo.

Os dados obtidos foram analisados com o auxílio do software Excel $^{\circledR}$ e Bioestat, versão 5.0. Inicialmente será feito tratamento descritivo dos dados por meio de média, desvio-padrão e variância dos domínios apresentados pelo teste citado acima (capacidade funcional, limitação por aspectos físicos, dor, estado geral de saúde, vitalidade, aspectos sociais, limitação por aspectos emocionais e saúde mental). Após realizar esse tratamento, no Bioestat, usando KruskalWallis com Dunnety pós-roc, avaliou-se a relação entre os domínios apresentados.

O estudo apresenta benefícios pois a avaliação da QV relacionada ao tratamento da DCR pode identificar aspectos que requerem maior atenção da equipe multiprofissional de saúde, dando subsídios para a implementação de novas estratégias e melhora da sobrevida dos pacientes.

Este trabalho foi submetido ao Comitê de Ética e Pesquisa com seres humanos e foi aprovado com o número 08279018.90000 .8041$.

\section{RESULTADOS}

Foram analisados os dados dos prontuários de 93 pacientes, 56 do sexo masculino (60\%) e 37 do sexo feminino (40\%). Em relação a etnia, 61 eram brancos (66\%), 18 eram pardos (19\%) e 14 eram negros (15\%). A média de idades ficou na casa dos 55 anos. Dos 93 pacientes abordados para entrevista, 2 se recusaram a responder o questionário e 91 o fizeram de maneira integral.

Tabela 1 - Sexo dos participantes da pesquisa. 2019.

\begin{tabular}{|c|c|c|}
\hline Sexo & Absoluta & Relativa \\
\hline Masculino & 56 & $60 \%$ \\
\hline Feminino & 37 & $40 \%$ \\
\hline Total & 93 & $100 \%$ \\
\hline
\end{tabular}


Tabela 2 - Cor da pele dos participantes da pesquisa. 2019.

\begin{tabular}{lcc}
\hline \multicolumn{1}{c}{ Cor/Raça } & Absoluta & Relativa \\
\hline Branca & 61 & $66 \%$ \\
Negra & 14 & $15 \%$ \\
Parda & 18 & $19 \%$ \\
Total & 93 & $100 \%$ \\
\hline & Fonte: os autores
\end{tabular}

As patologias de base foram agrupadas no Gráfico 1, sendo o Diabetes Mellitus (DM) a doença mais prevalente, estando presente em 39 dos 93 pacientes analisados: sendo que 37 pacientes possuíam DM de forma isolada e 2 possuíam DM e Hipertensão arterial Sistêmica (HAS). A HAS foi evidenciada em 28 pacientes: 24 pacientes possuíam HAS de forma isolada e 4 apresentavam outras patologias associadas. Outra patologia prevalente foi Glomerulonefrite Crônica, estando presente em 17 dos 93 pacientes analisados.

Gráfico 1 - Frequência Relativa das Doenças Crônicas. *Hiperplasia Prostática com Nefropatia Obstrutiva. *DM: Diabetes Mellitus. *HAS: Hipertensão Arterial Sistêmica.

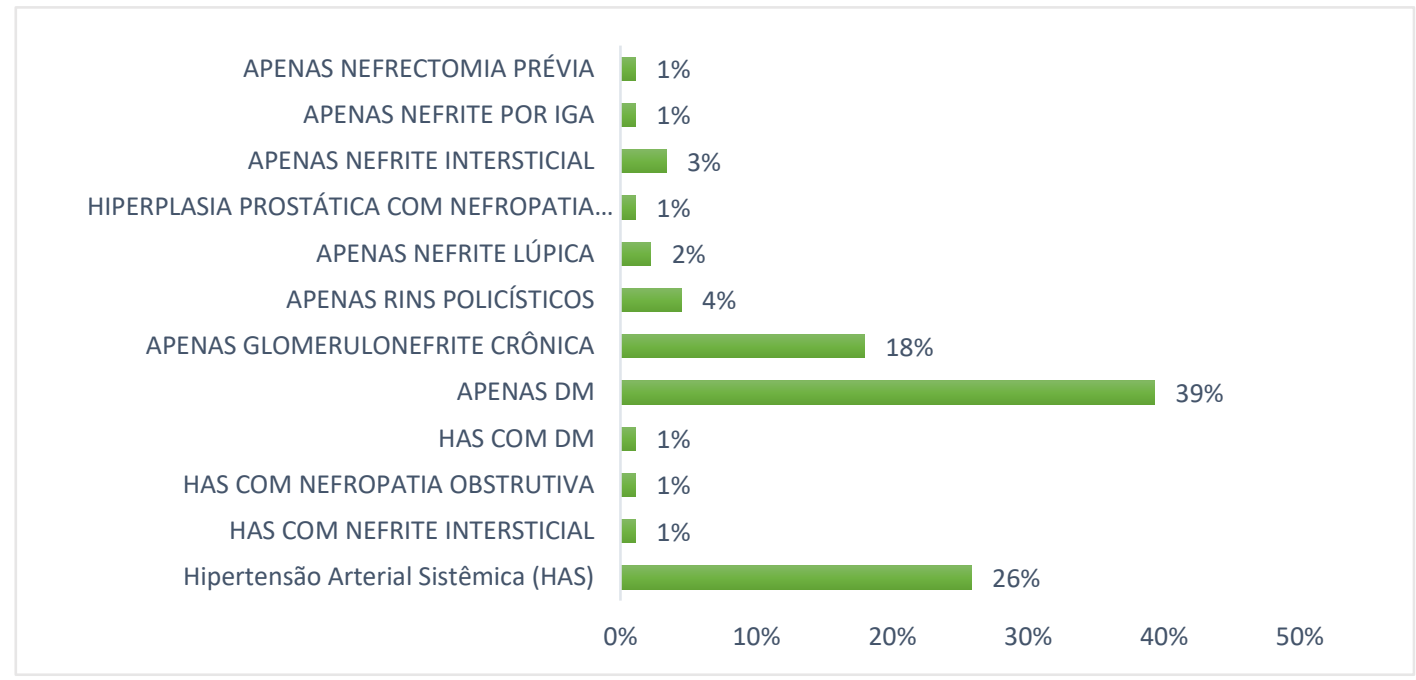

Fonte: os autores

O Quadro 3 agrupa os dados referentes renda, uso de antidepressivos (A.D.) e uso de terapia cognitivo comportamental (T.C.C.): a renda média foi de 1804 com desvio-padrão de 1601; 8 realizavam T.C.C e 83 não o faziam; 17 faziam uso de A.D e 74 não faziam uso de A.D.

Quadro 1 - Renda, Terapia Cognitivo-Comportamental (TCC) e Uso de Anti-depressivos (AD)

\begin{tabular}{|l|c|c|}
\hline \multicolumn{1}{|c|}{ Renda } & Média & $\mathbf{1 8 0 4}$ \\
\cline { 2 - 3 } & Desvio-Padrão & 1601 \\
\hline T.C.C. & Sim & 8 \\
\hline \multirow{2}{*}{ Uso de A.D. } & Não & 83 \\
\hline & Sim & 17 \\
\hline & Não & 74 \\
\hline
\end{tabular}

Os dados referentes à escolaridade são apresentados na tabela 3 mais da metade dos pacientes entrevistados apresentam ensino médio incompleto ou escolaridade mais baixa, $5,5 \%$ referem não ter estudado e $3,29 \%$ possuem ensino superior completo.

Tabela 3 - Grau de instrução dos participantes da pesquisa. 2019.

\begin{tabular}{lccc}
\hline \multicolumn{1}{c}{ Escolaridade } & N & $\%$ \\
\hline Ensino Fundamental Incompleto & 40 & 43,85 \\
\hline Ensino Fundamental Completo & 10 & 10,89 \\
\hline Ensino Médio Incompleto & 8 & 8,79 \\
\hline Ensino Médio Completo & 19 & 20,88 \\
\hline Ensino Superior Incompleto & 6 & 6,59 \\
\hline Ensino Superior Completo & 3 & 3,29 \\
\hline Não estudou & 5 & 5,5 \\
\hline TOTAL & 91 & 100 \\
\hline \multicolumn{1}{c}{ Fonte: os autores } & & \\
\hline
\end{tabular}

Os dados dos domínios da QV foram agrupados na tabela 4 em que se expõe as médias e os desviospadrões de cada domínio analisado. 0 domínio que 
apresentou a menor média foi "Limitações por aspectos físicos", com média de 40,88 de 100 pontos possíveis, enquanto a maior média foi visualizada em “Aspectos Sociais" com uma média de 79,56 de 100 pontos possíveis.

Tabela 4 - Relação dos domínios da qualidade de vida. 2019

\begin{tabular}{ccccccccc}
\hline DOMínIOS & $\begin{array}{c}\text { Capacidade } \\
\text { funcional }\end{array}$ & $\begin{array}{c}\text { Limitação } \\
\text { por aspectos } \\
\text { físicos }\end{array}$ & Dor & $\begin{array}{c}\text { Estado } \\
\text { geral de } \\
\text { saúde }\end{array}$ & Vitalidade & $\begin{array}{c}\text { Aspectos } \\
\text { sociais }\end{array}$ & $\begin{array}{c}\text { Aspectos } \\
\text { emocionais }\end{array}$ & $\begin{array}{c}\text { Saúde } \\
\text { Mental }\end{array}$ \\
\hline Média: & 56,48 & 40,88 & 63,66 & 50,41 & 65,44 & 79,56 & 56,07 & 72,8 \\
\hline Desvio Padrão & 30,91 & 41,95 & 28,61 & 22,88 & 24,4 & 27,83 & 44,4 & 25,13 \\
\hline \multicolumn{7}{c}{ Fonte: os autores }
\end{tabular}

Por se tratar de variáveis não-paramétricas e, portanto, com distribuição não normal, foi utilizado o teste de Kruskal-Wallis para avaliar a correlação entre os domínios.

A análise dos domínios "Capacidade Funcional", "Limitação por aspectos físicos", "Dor", "Estado geral de saúde", "Vitalidade", "Aspectos sociais", "Aspectos emocionais" e "Saúde mental" evidenciou um $\mathrm{KWH}=75,29$ com $\mathrm{p}<0,0001$.

A análise comparativa entre os domínios pelo método Dunn demonstrou um $\mathrm{p}<0,05$ para as comparações entre os seguintes domínios: Capacidade Funcional e Aspectos Sociais; Capacidade Funcional e Saúde Mental; Limitação por Aspectos Físicos e Dor; Limitação por Aspectos Físicos e Vitalidade; Limitação por Aspectos Físicos e Aspectos Sociais; Limitação por Aspectos Físicos e Saúde Mental; Dor e Aspectos Sociais; Estado geral de Saúde e Aspectos Sociais; Estado Geral de Saúde e Saúde Mental; Vitalidade e Aspectos Sociais.

Quadro 2 - Análise comparativa dos domínios (dunn $p<0,05)$

\begin{tabular}{|l|c|}
\hline \multicolumn{1}{|c|}{ Capacidade Funcional } & Aspectos Sociais \\
\hline Capacidade Funcional & Saúde Mental \\
\hline Limitações por Aspectos Físicos & Dor \\
\hline Limitações por Aspectos Físicos & Vitalidade \\
\hline Limitações por Aspectos Físicos & Aspectos Sociais \\
\hline Limitações por Aspectos Físicos & Saúde Mental \\
\hline Dor & Aspectos Sociais \\
\hline Estado Geral de Saúde & Aspectos Sociais \\
\hline Estado Geral de Saúde & Saúde Mental \\
\hline Vitalidade & Aspectos Sociais \\
\hline Aspectos Sociais & Aspectos Emocionais \\
\hline
\end{tabular}

$\mathrm{KWH}=\mathbf{7 5 , 2 9}$ com $\mathrm{p}<0,0001$ entre os domínios

Dados: $\mathrm{KWH}=75,29$ com $\mathrm{p}<0,0001$ entre os domínios

\section{DISCUSSÃO}

Conhecer o perfil epidemiológico dos pacientes que realizam hemodiálise na cidade de Araguari é importante por permitir conhecer a população submetida a esse tratamento e inferir sobre alguns aspectos da realidade psicossocial e da qualidade da assistência médica oferecida a essa população.

Os dados revelaram que a maioria dos pacientes são jovens (idade média de 55 anos) e predominantemente do sexo masculino, fato que se coaduna com o exposto no Censo 2017 da Sociedade Brasileira de Nefrologia, que indicava ser a maioria dos pacientes de idade jovem entre 45 a 64 anos, e com discreta predominância do sexo masculino.

A remuneração familiar média desses pacientes é baixa - apenas dois salários-mínimos e praticamente todos eles recebem auxílio da previdência social. A maioria desses pacientes origina-se de cidades vizinhas à cidade de Araguari e depende do transporte oferecido por suas prefeituras. Cabe ressaltar aqui a falta de investimentos públicos na abertura de novos Centros de Hemodiálise, sobretudo em cidades menores, o que acarreta dificuldades e desconforto aos pacientes que necessitam se deslocar para cidades vizinhas para realizar hemodiálise, permanecendo, muitas vezes, o dia todo fora de seus domicílios. Descentralização da distribuição dos centros de diálise no país constitui um problema de saúde pública, uma vez que o número de pacientes que realizam o processo aumentou em $159,4 \%$ no período de 2002 a 2017 (CENSO SBN, 2017).

Alia-se à remuneração familiar outro dado importante: os pacientes em hemodiálise têm significativamente menor escolaridade, o que evidencia que esses dois dados socioeconômicos estão associados ao risco de DRC.

Fonte: os autores 
A pobreza certamente influi na evolução dos pacientes que já fazem parte de Grupos de Risco, pois a educação deficiente, desnutrição, baixo peso ao nascer, déficit habitacional, alcoolismo, drogas, infecções crônicas, exposição à dejetos e poluentes ambientais e preconceito contribuem para que tenham sua doença básica agravada. (GORDAN, 2006, p. 10)

No que diz respeito às patologias de base, o $\mathrm{DM}$ e a HAS prevaleceram entre os pacientes analisados, o que se coaduna com os dados do Censo SBN 2017, evidenciando a estreita relação dessas patologias com a DRC. Como afirmam Júnior e Suassuna (2013, p. 53), cerca de $30 \%$ dos pacientes com diabetes mellitus desenvolvem nefropatia diabética, especialmente em associação com hipertensão arterial. Os autores asseveram, ainda, que "a doença renal crônica é um importante determinante de desfechos adversos em pacientes hipertensos e diabéticos, sendo considerada um dos mais significativos fatores não tradicionais de risco cardiovascular".

No que diz respeito ao quesito $Q V$, aqui definido em consonância com Buss (2000), como aspecto fundamental para se ter saúde, é imprescindível que existam políticas públicas amplas, efetivas, intersetoriais com vistas a uma boa QV. A deficiência em políticas públicas com visão multidimensional da qualidade de vida, empurra os indivíduos para quadros de comorbidades.

No caso da DRC não é diferente. O perfil sociodemográfico dos pacientes pesquisados evidencia que a pobreza contribui para o agravamento da doença. Os achados deste estudo, no que tange à $\mathrm{QV}$, demonstraram que a DRC interfere nos aspectos sociais, físicos, mentais e emocionais do paciente, repercutindo profundamente em sua vida, pois passa a viver exclusivamente "para a doença", deixando de lado sua vida ativa.

Esses achados vão ao encontro dos estudos realizados por Fassbinder et al (2014) em que os autores afirmam que "a doença renal crônica (DRC) interfere diretamente na capacidade funcional, na independência e, consequentemente, na qualidade de vida (QV)".

Os pacientes deste estudo responderam que o maior empecilho trazido pela doença se relaciona às limitações por aspectos físicos. As limitações físicas pesam muito mais para o paciente renal do que as mentais, sociais ou emocionais, pois repercutem na sua capacidade para o trabalho e atividades diárias.

Os dados deste estudo, revelados por meio do questionário aplicado aos pacientes com DRC, demonstram que estes, quando em tratamento dialítico, apresentam prejuízos em sua QV, pois veem reduzidas sua mobilidade cotidiana - como subir escadas - por exemplo, além de outros.

Assim, para além de uma visão clínica da doença, é preciso que os profissionais de saúde invistam no quesito $Q V$ do paciente com DRC a fim de reduzir suas limitações, promovendo sua autonomia.

\section{CONCLUSÃO}

O estudo demonstrou que a QV do paciente renal crônico, submetido à hemodiálise, sofre influências dos aspectos sociodemográficos e clínicos. Os aspectos físicos são os mais afetados.

A análise do perfil epidemiológico demonstrou que as dificuldades socioeconômicas constituem agravantes para os portadores de DRC.

Ao abordar essa temática e, ao analisar e cruzar os dados, fica claro para todos os componentes deste trabalho: A DRC cresce e crescerá virtuosamente com o aumento da pobreza. Em um país que não investe em saúde pública, que seus governantes lutam para aniquilar o único bem que garante a saúde do pobre - SUS - os profissionais de saúde, imbuídos de consciência humanizadora, devem voltar seu olhar para pesquisas que tragam melhorias para portadores de DRC e outras doenças crônicas.

As pesquisas podem trazer inovações capazes de melhorar o convívio do paciente renal com as limitações advindas de sua doença.

Além disso, investir também em educação em saúde e escolarização pode melhorar a QV do portador de DRC, pois propiciará maior conhecimento a respeito de sua doença, conduzirá ao autocuidado e evitará outras complicações.

\section{REFERÊNCIAS}

BORTOLOTTO, Luiz Aparecido. Hipertensão arterial e insuficiência renal crônica. Revista Brasileira de Hipertensão, v. 15, n. 3, p. 152-5, 2008.

BUSS, P. M. Promoção da saúde e qualidade de vida. Ciência \& Saúde Coletiva, Rio de Janeiro, v.5, n.1, p.163-77, 2000.

CAVALCANTE, Francielly Almeida et al. O uso do Iúdico em hemodiálise: buscando novas perspectivas na qualidade de atendimento ao paciente no centro 
de diálise. Revista Eletrônica da Facimed, v. 3, n. 3, p. 371-384, 2011.

CRAVO, Carla Daniele Lopes et al. Perfil epidemiológico dos pacientes em hemodiálise de um hospital universitário. Ciências Cuidado e Saúde, v. 10, n. 1, p. 110-5, 2011.

FRAZÃO, Cecília Maria Farias de Queiroz; RAMOS, Vânia Pinheiro; LIRA, Ana Luisa Brandão de Carvalho. Qualidade de vida de pacientes submetidos a hemodiálise. Revista de Enfermagem. UERJ, v. 19, n. 4, p. 577-582, 2011.

FASSBINDER et al. Capacidade funcional e qualidade de vida de pacientes com doença renal crônica, prédialítica e em hemodiálise - um estudo transversal. Jornal Brasileiro de Nefrologia, 2015;37(1):47-54

GIL, Antônio Carlos. Métodos e técnicas de pesquisa social. 6.ed. São Paulo: Atlas, 2008.

GORDAN, Pedro. A. Grupos de Risco para doença renal crônica. Jornal Brasileiro de Nefrologia. Volume XXVIII - no 3 - Supl. 2 - Setembro de 2006.

HIGA, Karina et al. Qualidade de vida de pacientes portadores de insuficiência renal crônica em tratamento de hemodiálise. Acta Paulista de Enfermagem, v. 21, 2007.

JÚNIOR, José Mauro Vieira. SUASSUNA. José Hermógenes Rocco. O acometimento renal na hipertensão arterial e diabetes mellitus tipo 2: como identificar e prevenir A visão do nefrologista. Revista HUPE, Rio de Janeiro, 2013;12(Supl 1):53-60.

KUSUMOTO, Luciana et al. Adultos e idosos em hemodiálise: avaliação da qualidade de vida relacionada à saúde. Acta Paulista de Enfermagem, v. 21, 2008.

MARTINS, Marielza R. Ismael; CESARINO, Claudia Bernardi. Qualidade de vida de pessoas com doença renal crônica em tratamento hemodialítico. Revista Latino-Americana de Enfermagem, v. 13, n. 5, p. 670-676, 2005.

MOREIRA, Humberto Graner et al. Diabetes mellitus, hipertensão arterial e doença renal crônica: estratégias terapêuticas e suas limitações. Revista Brasileira de Hipertensão, v. 15, n. 2, p. 111-6, 2008.

SCHWARTZ, Eda et al. Caracterização dos serviços de terapia renal substitutiva da metade sul do Rio
Grande do Sul/Brasil. Revista Latino-Americana de Enfermagem, v. 20, n. 2, p. 86-98, 2017

Sociedade Brasileira de Nefrologia.[internet].Censo de Diálise SBN 2017. Disponível em: https://sbn.org.br/categoria/censo-2017/. Acesso em 2018/2019.

SODRÉ, Fábio L.; COSTA, Josete Conceição Barreto; LIMA, José Carlos C. "Avaliação da função e da lesão renal: um desafio laboratorial." Jornal Brasileiro de Patologia e Medicina Laboratorial 43.5 (2007): 329337.

TRENTINI, Mercedes et al. Qualidade de vida de pessoas dependentes de hemodiálise considerando alguns aspectos físicos, sociais e emocionais. Texto \& Contexto Enfermagem, v. 13, n. 1, 2004.

VIEIRA CASSINI, Amanda et al. Avaliação dos principais fatores etiológicos em indivíduos portadores de insuficiência renal crônica em hemodiálise. Conscientia e Saúde, v. 9, n. 3, 2010. 